\title{
The association of smoking status with SARS-CoV-2 infection, hospitalisation and mortality from COVID-19: A living rapid evidence review
}

\author{
David Simons ${ }^{1}$, Lion Shahab², Jamie Brown², Olga Perski \\ 1 Royal Veterinary College, RVC \\ 2 Univers ity College London, University of London
}

\begin{abstract}
Background: SARS-CoV-2 is the causative agent of COVID-19, an emergent zoonotic disease which has reached pandemic levels and is designated a public health emergency of international concern. It is plausible that former or current smoking status are risk factors for infection, hospitalisation and/or mortality from COVID-19.
\end{abstract}

Objective: We aimed to estimate the rates of i) infection, ii) hospitalisation, iii) disease severity, and iv) mortality from SARS-CoV-2/COVID-19 stratified by smoking status.

Methods: We adopted recommended practice for rapid evidence reviews, which involved limiting the search to main databases and having one reviewer extract data and another verify. Published articles and pre-prints were identified via MEDLINE, EPPIMapper and expertise within the review team. We included observational studies with community-dwelling or hospitalised adults aged $16+$ years who had been tested for SARS-CoV-2 or were diagnosed with COVID-19, providing that data on smoking status were reported. Studies were judged as high quality if they explicitly recorded current, former and never smoking status with low levels of missing data.

Results: T wenty-eight studies were included, 22 of which were conducted in China, three in the US, one in Korea, one in France and one across multiple international sites with data predominantly collected in the UK. Eight studies did not state the source for information on smoking status. Twenty-five studies reported current and/or former smoking status but had high levels of missing data and/or did not explicitly state whether the remaining participants were never smokers. Notwithstanding these uncertainties, compared with national prevalence estimates, recorded current and 
former smoking rates in the included studies were generally lower than expected. Within the only study to report community SARS-CoV-2 infection by smoking status, current smokers appeared more likely to be tested but the rate for positive tests was lower. In two high-quality studies, results from a fixed-effects meta-analysis provided no evidence for an increased risk of hospitalisation among 657 current/former smokers who tested positive in the community $(R R=1.03,95 \% \mathrm{Cl}=0.93-1.14, \mathrm{p}=$ 0.57). Among 1370 people hospitalised across two high-quality studies, there was greater disease severity in current/former smokers compared with never smokers (RR $=1.43,95 \% \mathrm{Cl}=1.15-1.77, \mathrm{p}=.002)$. Three studies reporting on mortality did not explicitly state never smoking status.

Conclusions: Across 28 observational studies, there is substantial uncertainty arising from the recording of smoking status on whether current and/or former smoking status is associated with SARS-CoV-2 infection, hospitalisation or mortality. There is low quality evidence that current and former smoking compared with never is associated with greater disease severity in those hospitalised for COVID-19.

Implications: Unrelated to COVID-19, smokers are at a greater risk of a range of serious health problems requiring them to be admitted to hospital. Given uncertainty around the association of smoking with COVID-19, smoking cessation remains a public health priority and high-quality smoking cessation advice should form part of public health efforts during this pandemic.

\section{Introduction}

COVID-19 is a respiratory disease caused by the emerging SARS-CoV-2 virus. Large age and gender differences in case severity and mortality have been observed in the ongoing COVID-19 pandemic ${ }^{[1]}$; however, these differences are currently unexplained. SARS-CoV2 enters epithelial cells through the ACE2 receptor ${ }^{[2]}$. Some evidence suggests that gene expression and subsequent receptor levels are elevated in the airway and oral epithelium of current smokers ${ }^{[3][4]}$, thus putting smokers at higher risk of contracting SARS-CoV-2. Other studies, however, show that nicotine downregulates the ACE2 receptor ${ }^{[5]}$. These uncertainties notwithstanding, both former and current smoking is known to increase the risk of respiratory vira[ ${ }^{[6][7]}$ and bacterial[ ${ }^{[8][9]}$ infections and is associated with worse outcomes once infected. Cigarette smoke reduces the respiratory immune defence through peri-bronchiolar inflammation and fibrosis, impaired mucociliary clearance and 
disruption of the respiratory epithelium ${ }^{[10]}$. There is also reason to believe that behavioural factors (e.g. regular hand-to-mouth movements) involved in smoking may increase SARS-CoV-2 infection and transmission in current smokers. However, early data from the COVID-19 pandemic have not provided clear evidence for a negative impact of former and/or current smoking on SARS-CoV-2 infection or COVID-19 related outcomes, such as hospitalisation or mortality ${ }^{[11]}$.

There are several reviews that fall within the scope of smoking and COVID$19^{[12][13][14][15][16]}$. We aimed to produce a rapid synthesis of available evidence pertaining to the rates of infection, hospitalisation, disease severity and mortality from SARS-CoV-2/COVID-19 stratified by smoking status. Given the rapidly increasing availability of data on this topic, this will be a 'living' review with regular updates.

\section{Methods}

\section{Study design}

We adopted recommended practice for rapid evidence reviews, which involved limiting the search to main databases and having one reviewer extract the data and another verify $^{[17]}$.

\section{Eligibility criteria}

Studies were included if they:

1) Were primary research studies using experimental (e.g. randomised controlled trial), quasi-experimental (e.g. pre- and post-test) or observational (e.g. case-control) study designs;

2) Involved as participants adults aged 16+ years;

3) Recorded as outcome i) results of a SARS-CoV-2 diagnostic assay, ii) a clinical diagnosis of COVID-19, iii) hospitalisation for COVID-19, iv) severity of COVID-19 disease or v) mortality from COVID-19;

4) Reported any of the outcomes of interest by self-reported or biochemically verified smoking status (e.g. current smoker, former smoker, never smoker);

5) Were written in English;

6) Were published in a peer-reviewed journal, as a pre-print or a public health report by reputable agents (e.g. governments, scientific societies). 
Studies were excluded if they:

1) Were published as a conference abstract.

\section{Search strategy}

We identified articles through setting up an alert on Ovid MEDLINE, searching for the terms 'smoking' and 'COVID-19' in titles, abstracts and as key words. We also screened studies listed under 'T ransmission/risk/prevalence' in EPPI-Mapper, a living map of published evidence related to COVID-19. Pre-prints and public health reports were identified through social media (e.g. Twitter) and expertise within the review team.

\section{Selection of studies}

One reviewer screened titles, abstracts and full texts ag ainst the inclusion criteria.

\section{Data extraction}

Data were extracted by one and verified by a second reviewer on i) author (year); ii) date published; iii) country; iv) study design; v) setting; vi) sample size; vii) sex; viii) age; ix) smoking status (e.g. current, former, never, missing); x) SARS-CoV-2 infection; xi) diagnosis of COVID-19; xii) hospitalisation for COVID-19; xiii) disease severity; and xiv) mortality.

\section{Quality appraisal}

Studies were judged as high quality if they explicitly recorded current, former and never smoking status with low levels of missing data.

\section{Evidence synthesis}

A narrative synthesis was conducted. Where possible, data were pooled in RevMan v.5.3 $3^{[18]}$ with the Mantel-Haenzel method using random or fixed effects, depending on heterogeneity, and presented as risk ratios (RRs) ${ }^{[19]}$. Heterogeneity between study outcomes was assessed using the $\mathrm{I}^{2}$ statistic, suitable for smaller meta-analyses ${ }^{[20]}$. 
Results

A total of 310 records were identified, of which 75 full texts were screened and 28 studies were included in the narrative synthesis (see Figure 1).

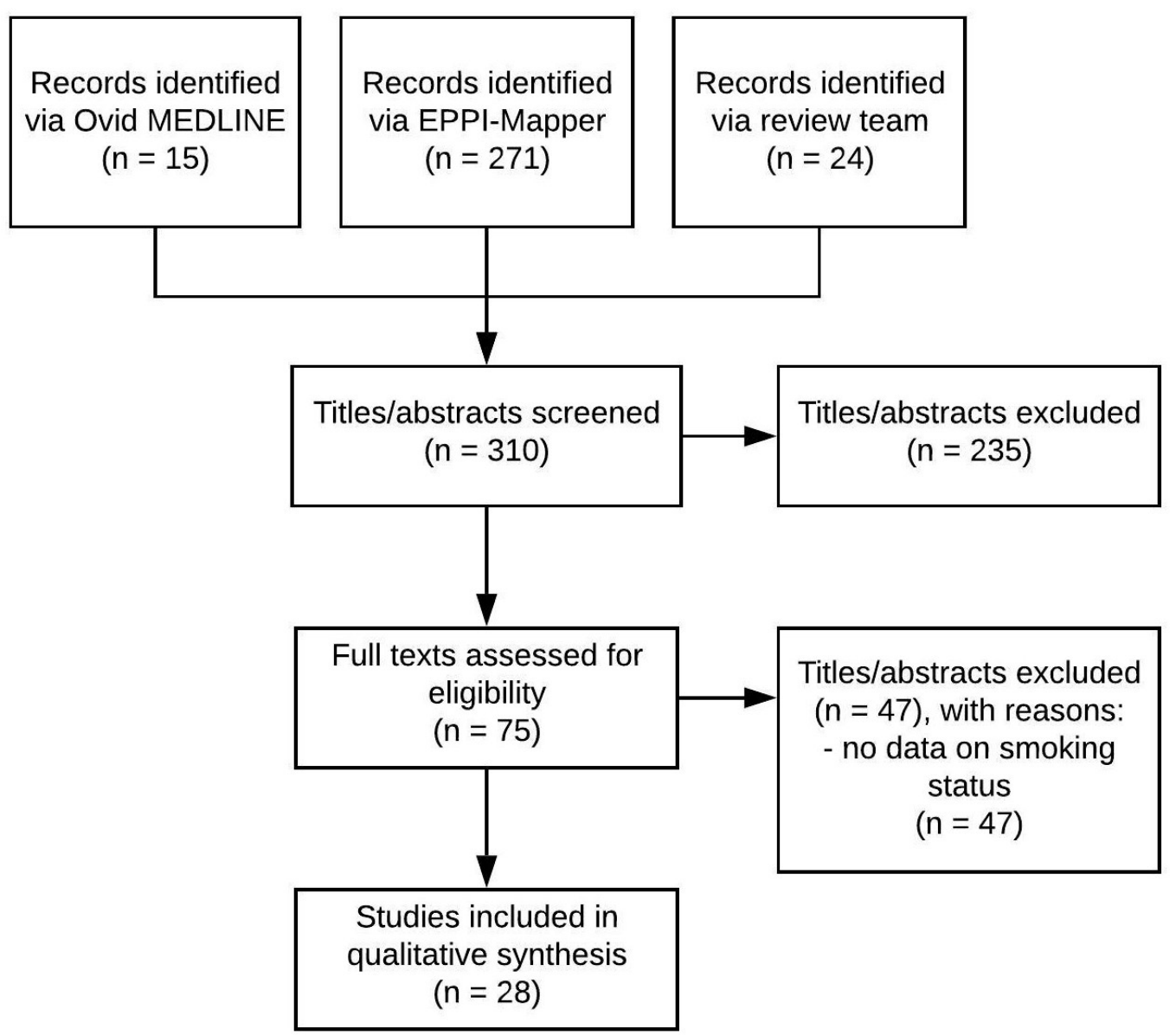

Figure 1. PRISMA flow diagram of included studies

\section{Study characteristics}

Characteristics of included studies are presented in Table 1. Twenty-two studies were conducted in China[1][21][22][23][24][25][26][27][28][29][30][31][32][33][34][35][36][37], three in the US $^{[38][39][40]}$, one in Korea ${ }^{[41]}$, one in France ${ }^{[42]}$ and one multi-site international study with the majority of participants $(82.1 \%)$ being recruited in the UK ${ }^{[43]}$. Twenty-four studies were conducted in hospital settings. Four studies included a community component in addition to hospitalised patients[38][39][40][42] . Sample sizes ranged from 9 to 7,162. The total sample analysed in the current review was 23,067 participants. 


\section{Data quality}

The levels used to categorise smoking status were heterogeneous (see Table 1). Notably, only three studies recorded current, former and never smoking status, with 25 studies reporting current and/or former smoking status but did not explicitly state whether the remaining participants were never smokers or whether data on smoking status were missing (labelled 'never smoker/unknown' throughout). Smoking status was predominantly collected through routine electronic health records. Six studies used a bespoke case report form for COVID-19 ${ }^{[32][37][42][44][45][46]}$. None of the studies verified smoking status biochemically. Two studies ${ }^{[33][46]}$ specifically stated that smokers were those with a >30 pack-year history or a greater than 20-year history of smoking, respectively. Most studies did not assess tobacco exposure (e.g. pack-years of smoking) in current or former smokers, or time since quitting in former smokers.

\section{Smoking prevalence among those with a COVID-19 diagnosis by country}

In the included studies conducted in hospital settings across China, $3.8 \%$ to $17.6 \%$ were current smokers and $1.9 \%$ to $5.0 \%$ were former smokers. However, current and former smoking prevalence in China was reported to be $50.5 \%$ and $8.4 \%$ respectively among men and $2.1 \%$ and $0.8 \%$ among women in $2018^{[47]}$, thus suggesting lower than expected proportions of current and former smokers in the included studies.

In the included studies conducted in the US across community and hospital settings, 1.3\% to $27.2 \%$ were current smokers and $2.3 \%$ to $30.6 \%$ were former smokers. This compares with a smoking prevalence of $13.8 \%$ current and $20.9 \%$ former smokers in the US in $2018^{[48]}$.

In the included study conducted in Korea from a hospital setting, $18.5 \%$ were current smokers. This compares with a national smoking prevalence of $19.3 \%$ in Korea in $2016^{[49]}$.

In the included study conducted in France, 7.1\% were current smokers and 59.1\% were former smokers across an outpatient and inpatient hospital setting, with $6.2 \%$ and $61.0 \%$ among those hospitalised. This compares with a current and former smoking prevalence of $32.0 \%$ and $31.4 \%$ respectively in France in $2018^{[50]}$, thus sugg esting a lower than 
expected proportion of current smokers in the included study but higher than expected proportion of former.

In the multi-site study with participants predominantly from the UK in a hospital setting, $5.0 \%$ were current or former smokers. This compares with a current and former smoking prevalence of $14.4 \%$ and $25.8 \%$ in England in $2018^{[51]}$, thus suggesting a lower than expected proportion of current and former smokers in the included study.

\begin{tabular}{|c|c|c|c|c|c|c|c|c|}
\hline Author & Date published & Country & Sample size & Setting & $\begin{array}{l}\text { Median age } \\
\text { (IQR) }\end{array}$ & \% Female & Smoking status of those COVID+ & $\begin{array}{l}\text { Data source for smoking } \\
\text { status }\end{array}$ \\
\hline Guan, $\mathrm{Ni}$ & $28 / 02 / 2020$ & China & 1099 & Hospital & $47(35-58)$ & $41.9 \%$ & $\begin{array}{l}\text { Current smoker }(12.6 \%) \\
\text { Forrer smoker }(1.9 \%) \\
\text { Never smoker }(85.4 \%) \\
\text { Missing }(1.3 \%)\end{array}$ & Not stated \\
\hline Guan, Liang & $26 / 03 / 2020$ & China & 1590 & Hospital & $49(33-64)$ & $42.7 \%$ & $\begin{array}{l}\text { Current/former smoker (7.0\%) } \\
\text { Never smoker }(93.0 \%)\end{array}$ & Not stated \\
\hline Lian & $25 / 03 / 2020$ & China & 788 & Hospital & $\therefore$ & $38.5 \%$ & $\begin{array}{l}\text { Current smoker (6.9\%) } \\
\text { Not stated (93.1\%) }\end{array}$ & Not stated \\
\hline Jin & $24 / 03 / 2020$ & China & 651 & Hospital & $46(32-60)$ & $49.2 \%$ & $\begin{array}{l}\text { Current smoker }(6.3 \%) \\
\text { Not stated }(93.7 \%)\end{array}$ & Not stated \\
\hline Chen & $26 / 03 / 2020$ & China & 548 & Hospital & $62(44-70)$ & $37.6 \%$ & $\begin{array}{l}\text { Current smoker }(4.4 \%)^{*} \\
\text { Former smoker }(2.6 \%)^{*} \\
\text { Not stated }(93.1 \%){ }^{2}\end{array}$ & Not stated \\
\hline Zhou & $11 / 03 / 2020$ & China & 191 & Hospital & $56(46-67)$ & $38.0 \%$ & $\begin{array}{l}\text { Current smoker (6.0\%) } \\
\text { Not stated }(94.0 \%)\end{array}$ & Not stated \\
\hline Mo & $16 / 03 / 2020$ & China & 155 & Hospital & $54(53-66)$ & $44.5 \%$ & $\begin{array}{l}\text { Current smoker }(3.9 \%) \\
\text { Not stated }(96.1 \%)\end{array}$ & Case report form \\
\hline Zhang, Dong & $19 / 02 / 2020$ & China & 140 & Hospital & $57(25-87)^{\wedge}$ & $46.3 \%$ & $\begin{array}{l}\text { Current smoker }(1.4 \%) \\
\text { Former smoker }(5.0 \%) \\
\text { Not stated }(93.6 \%)\end{array}$ & Electronic health records \\
\hline Wan & $21 / 03 / 2020$ & China & 135 & Hospital & $47(36-55)$ & $46.7 \%$ & $\begin{array}{l}\text { Current smoker (6.7\%) } \\
\text { Not stated }(93.3 \%)\end{array}$ & Electronic health records \\
\hline Liu, Tao & $28 / 02 / 2020$ & China & 78 & Hospital & $38(33-57)$ & $50.0 \%$ & $\begin{array}{l}\text { Current/former smoker (6.4\%) } \\
\text { Not stated (93.6\%) }\end{array}$ & Case report form \\
\hline Huang, Wang & $05 / 03 / 2020$ & China & 41 & Hospital & $49(41-58)$ & $27.0 \%$ & $\begin{array}{l}\text { Current smoker }(7.3 \%) \\
\text { Not stated }(92.7 \%)\end{array}$ & Electronic health records \\
\hline Zhang, Cai & $20 / 03 / 2020$ & China & 645 & Hospital & $\therefore$ & $49.1 \%$ & $\begin{array}{l}\text { Current smoker (6.4\%) } \\
\text { Not stated }(93.5 \%)\end{array}$ & Electronic health records \\
\hline Guo & $27 / 03 / 2020$ & China & 187 & Hospital & $59(45-73)$ & $51.3 \%$ & $\begin{array}{l}\text { Current smoker }(9.6 \%) \\
\text { Not stated }(90.4 \%)\end{array}$ & Electronic health records \\
\hline Liu, Ming & $12 / 03 / 2020$ & China & 41 & Hospital & $39(30-48)$ & $58.5 \%$ & $\begin{array}{l}\text { Current smoker }(9.8 \%) \\
\text { Not stated }(90.2 \%)\end{array}$ & Electronic health records \\
\hline Huang, Yang & 05/03/2020 & China & 36 & Hospital & $69(60-78)$ & $30.6 \%$ & $\begin{array}{l}\text { Current/former smoker (11.1\%) } \\
\text { Not stated }(88.9 \%)\end{array}$ & Not stated \\
\hline
\end{tabular}

\begin{tabular}{|c|c|c|c|c|c|c|c|c|}
\hline Author & $\begin{array}{l}\text { Date } \\
\text { published }\end{array}$ & Country & $\begin{array}{l}\text { Sample } \\
\text { size }\end{array}$ & Setting & $\begin{array}{l}\text { Median age } \\
\text { (IQR) }\end{array}$ & \% Female & Smoking status of those COVID+ & $\begin{array}{l}\text { Data source for smoking } \\
\text { status }\end{array}$ \\
\hline Xu & $08 / 03 / 2020$ & China & 53 & Hospital & $\because$ & $47.2 \%$ & $\begin{array}{l}\text { Current smoker }(11.3 \%) \\
\text { Not stated }(88.7 \%)\end{array}$ & Electronic health records \\
\hline Li & $12 / 02 / 2020$ & China & 17 & Hospital & $45(33-57)$ & $47.1 \%$ & $\begin{array}{l}\text { Current smoker (17.6\%) } \\
\text { Not stated (82.4\%) }\end{array}$ & Electronic health records \\
\hline Rentsch $\sim$ & $14 / 04 / 2020$ & USA & 3789 & Community/hospital & $66(60-70)$ & $4.6 \%$ & $\begin{array}{l}\text { Current smoker (27.2\%) } \\
\text { Former smoker (30.6\%) } \\
\text { Never smoker (36.9\%) } \\
\text { Missing (5.3\%) }\end{array}$ & Electronic health records \\
\hline Hu & $25 / 03 / 2020$ & China & 323 & Hospital & $61(23-91)^{\wedge}$ & $48.6 \%$ & $\begin{array}{l}\text { Current/former smoker }(11.8 \%) \\
\text { Not stated }(88.2 \%)\end{array}$ & Not stated \\
\hline Wang & $24 / 03 / 2020$ & China & 125 & Hospital & $41(26-66)$ & $43.2 \%$ & $\begin{array}{l}\text { Current/former smoker }(11.8 \%) \\
\text { Not stated }(87.2 \%)\end{array}$ & Electronic health records \\
\hline ISARIC & 08/04/2020 & Multiple & 3316 & Hospital & $71(0-104)^{\wedge}$ & $37.0 \%$ & $\begin{array}{l}\text { Current/former smoker (5.0\%) } \\
\text { Never smoker (42.0\%) } \\
\text { Missing (54.0\%) }\end{array}$ & Case report form \\
\hline Petrilli & $11 / 04 / 2020$ & USA & 4103 & Community/hospital & $52(36-65)$ & $47.9 \%$ & $\begin{array}{l}\text { Current smoker }(5.2 \%) \\
\text { Former smoker }(16.2 \%) \\
\text { Never smoker/unknown }(78.6 \%)\end{array}$ & Electronic health records \\
\hline $\begin{array}{l}\text { CDC COVID-19 Response } \\
\text { Team }\end{array}$ & $31 / 03 / 2020$ & USA & 7162 & Community/hospital & $\therefore$ & - & $\begin{array}{l}\text { Current smoker }(1.3 \%) \\
\text { Former smoker }(2.3 \%) \\
\text { Missing }(96.4 \%)\end{array}$ & Electronic health records \\
\hline Miyara & $21 / 04 / 2020$ & France & 482 & Community/hospital & $\therefore$ & $43.0 \%$ & $\begin{array}{l}\text { Current/occasional smoker } \\
(7.1 \%) \\
\text { Former smoker }(59.1 \%) \\
\text { Never smoker }(32 \%) \\
\text { Missing }(1.8 \%)\end{array}$ & Case report form \\
\hline Dong & $20 / 03 / 2020$ & China & 9 & Hospital & $44(30-46)$ & $66.7 \%$ & $\begin{array}{l}\text { Current smoker (11.1\%)\# } \\
\text { Never smoker/unknown (88.9\%) }\end{array}$ & Electronic health records \\
\hline Kim & $01 / 04 / 2020$ & Korea & 28 & Hospital & $43(30-56)$ & $46.4 \%$ & $\begin{array}{l}\text { Current smoker (18.5\%) } \\
\text { Never smoker/unknown ( } 81.5 \%)\end{array}$ & Electronic health records \\
\hline Shi & $18 / 03 / 2020$ & China & 487 & Hospital & $46(27-65)$ & $46.8 \%$ & $\begin{array}{l}\text { Current/former smoker }(8.2 \%) \\
\text { Never smoker/unknown }(89.1 \%)\end{array}$ & Case report form \\
\hline Yang & $24 / 02 / 2020$ & China & 52 & Hospital & $60(47-73)$ & $37.0 \%$ & $\begin{array}{l}\text { Current smoker (3.8\%) } \\
\text { Never smoker/unknown }(96.2 \%)\end{array}$ & Case report form \\
\hline
\end{tabular}




\section{SARS-CoV-2 infection by smoking status}

One study provided data on SARS-CoV-2 infection for people meeting local testing criteria, stratified by smoking status (see T able 2). Data were obtained from a cohort study of US military veterans. Current smokers were more likely to receive a test: $42.3 \%$ (1603/3789) of the sample were current smokers compared with $21.6 \%$ of all veterans over 18 between $2010-2015^{[52]}(z=25.52, p<.001)$. However, individuals with a smoking history (i.e. current/former smokers) appeared less likely to test positive for SARS-CoV-2 than never smokers: of those with negative tests, $72.2 \%$ were current/former smokers. This compares with $61.3 \%$ of those with positive tests being current/former smokers.

\section{Hospitalisation for COVID-19 by smoking status}

Three studies assessed hospitalisation for COVID-19 disease stratified by smoking status (see Table 3). A fixed-effects meta-analysis was performed for two studies in which never smoking status was ascertained (as opposed to grouping never smokers with those with unknown status). There was no significant difference between current/former smokers and never smokers in the risk of requiring admission to hospital $(R R=1.03,95 \%$ $\mathrm{Cl}=0.93-1.14, \mathrm{p}=0.57$; see Figure 2).

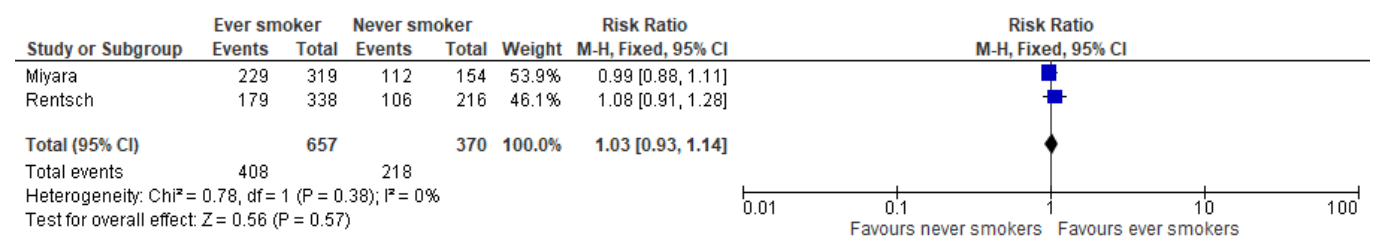

Figure 2. Forest plot for risk of hospitalisation by smoking status.

\section{Disease severity by smoking status}

Ten studies reported disease severity stratified by smoking status (see Table 4). Severe disease (as opposed to non-severe disease) was broadly defined across studies as requiring ITU admission, requiring oxygen as a hospital inpatient or in-hospital death (where this had not been disagg regated into disease severity vs. mortality). A fixedeffects meta-analysis was performed for two studies in which never smoking status was ascertained (as opposed to grouping never smokers with those with unknown status), 
indicating that smokers were of greater risk of experiencing severe disease compared with never smokers ( $R R=1.43,95 \% C l=1.15-1.77, p=.002$; see Figure 3 ).

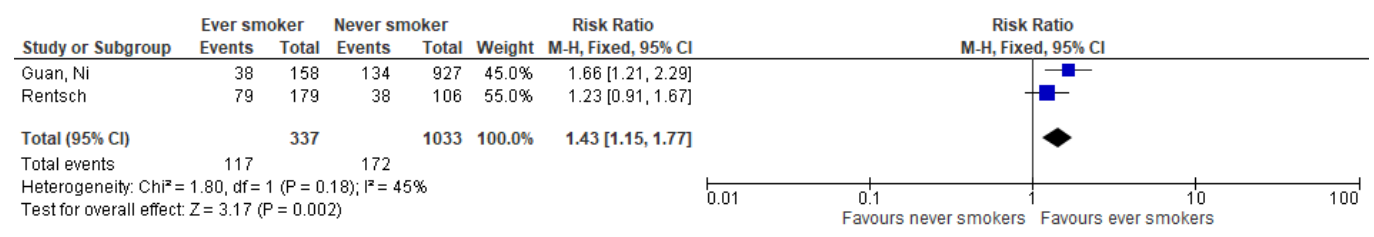

Figure 3. Forest plot for the risk of severe disease by smoking status

\section{Mortality by smoking status}

Three studies reported mortality from COVID-19 stratified by smoking status (see Table 5). As all three studies had potentially high levels of missing data (i.e. $>90 \%$ of individuals with no stated smoking history), a meta-analysis was not performed. There did not appear to be a notable difference between current/former smokers and never smokers/unknown status in mortality.

Table 2. SARS-CoV-2 infection by smoking status.

\begin{tabular}{|c|c|c|c|c|c|c|c|c|c|}
\hline & $\begin{array}{l}\text { Total population } \\
\text { tested }\end{array}$ & $\begin{array}{l}\text { SARS-CoV-2 } \\
\text { negative }\end{array}$ & & & & $\begin{array}{l}\text { SARS-CoV-2 } \\
\text { positive }\end{array}$ & & & \\
\hline Author & $\mathrm{N}$ & $\mathrm{N}$ & $\begin{array}{l}\text { Current } \\
\text { smoker }\end{array}$ & $\begin{array}{l}\text { Former } \\
\text { smoker }\end{array}$ & $\begin{array}{l}\text { Never } \\
\text { smoker }\end{array}$ & $\mathrm{N}$ & $\begin{array}{l}\text { Current } \\
\text { smoker }\end{array}$ & $\begin{array}{l}\text { Former } \\
\text { smoker }\end{array}$ & $\begin{array}{l}\text { Never } \\
\text { smoker }\end{array}$ \\
\hline Rentsch & $3528^{*}$ & $2974^{*}$ & $1444(48.6 \%)$ & $704(23.6 \%)$ & $826(27.8 \%)$ & $554^{*}$ & $159(28.7 \%)$ & $179(32.3 \%)$ & 216 (39.0\%) \\
\hline
\end{tabular}

Note. ${ }^{*}$ Data on smoking status were missing for 261 participants

Table 3. Hospitalisation for COVID-19 by smoking status.

\begin{tabular}{|c|c|c|c|c|c|c|c|c|c|c|c|}
\hline & COVID tve & Community & & & & & Hospitalised & & & & \\
\hline Author & $\mathrm{N}$ & $\mathrm{N}$ & $\begin{array}{l}\text { Current } \\
\text { smoker }\end{array}$ & $\begin{array}{l}\text { Former } \\
\text { smoker }\end{array}$ & $\begin{array}{l}\text { Never } \\
\text { smoker }\end{array}$ & Never/unknown & $\mathrm{N}$ & $\begin{array}{l}\text { Current } \\
\text { smoker }\end{array}$ & $\begin{array}{l}\text { Former } \\
\text { smoker }\end{array}$ & $\begin{array}{l}\text { Never } \\
\text { smoker }\end{array}$ & Never/unknown \\
\hline Rentsch & $554^{*}$ & $269(48.6 \%)$ & $69(25.7 \%)$ & $90(33.5 \%)$ & $\begin{array}{l}110 \\
(40.8 \%)\end{array}$ & - & 285 (51.4\%) & $90(31.6 \%)$ & $89(31.2 \%)$ & $\begin{array}{l}106 \\
(37.2 \%)\end{array}$ & - \\
\hline Petrilli & 4103 & $\begin{array}{l}2104 \\
(51.3 \%)\end{array}$ & $108(5.1 \%)$ & $250(11.9 \%)$ & - & 1746 (83.0\%) & $\begin{array}{l}1999 \\
(48.7 \%)\end{array}$ & $104(5.2 \%)$ & 416 (20.8\%) & - & $1479(74.0 \%)$ \\
\hline Miyara & $482^{* *}$ & 139 (28.8\%) & $13(9.4 \%)$ & $77(55.4 \%)$ & $42(30.2 \%)$ & - & 343 (71.1\%) & $21(6.1 \%)$ & $208(60.6 \%)$ & $\begin{array}{l}112 \\
(32.7 \%)\end{array}$ & - \\
\hline
\end{tabular}

Note. * Data on smoking status were missing for 31 participants; ** Data on smoking status were missing for 9 participants. 
Table 4. Disease severity by smoking status.

\begin{tabular}{|c|c|c|c|c|c|c|c|c|c|c|c|c|c|}
\hline & $\begin{array}{l}\text { Sample } \\
\text { size }\end{array}$ & $\begin{array}{l}\text { Non- } \\
\text { severe } \\
\text { disease }\end{array}$ & & & & & & $\begin{array}{l}\text { Severe } \\
\text { disease }\end{array}$ & & & & & \\
\hline Author & $\mathrm{N}$ & $\mathrm{n}$ & $\begin{array}{l}\text { Current } \\
\text { smoker }\end{array}$ & $\begin{array}{l}\text { Former } \\
\text { smoker }\end{array}$ & $\begin{array}{l}\text { Current/former } \\
\text { smoker }\end{array}$ & $\begin{array}{l}\text { Never } \\
\text { smoker }\end{array}$ & $\begin{array}{l}\text { Never } \\
\text { smoker/unknown }\end{array}$ & $\mathrm{n}$ & $\begin{array}{l}\text { Current } \\
\text { smoker }\end{array}$ & $\begin{array}{l}\text { Former } \\
\text { smoker }\end{array}$ & $\begin{array}{l}\text { Current/former } \\
\text { smoker }\end{array}$ & $\begin{array}{l}\text { Never } \\
\text { smoker }\end{array}$ & $\begin{array}{l}\text { Never } \\
\text { smoker/unknown }\end{array}$ \\
\hline Guan, Ni & $1085^{\circ}$ & $\begin{array}{l}913 \\
(84.1 \%)\end{array}$ & $\begin{array}{l}108 \\
(11.8 \%)\end{array}$ & $12(1.3 \%)$ & & $\begin{array}{l}793 \\
(86.9 \%)\end{array}$ & . & $\begin{array}{l}172 \\
(15.9 \%)\end{array}$ & $\begin{array}{l}29 \\
(16.9 \%]\end{array}$ & $9(5.2 \%)$ & - & $\begin{array}{l}134 \\
(77.9 \%)\end{array}$ & \\
\hline $\begin{array}{l}\text { Zhang, } \\
\text { Dong, }\end{array}$ & $9^{b}$ & $3(33.3 \%)$ & $0(0.0 \%)$ & $\begin{array}{l}3 \\
\text { (100.0\%) }\end{array}$ & - & - & - & $\begin{array}{l}6 \\
(66.7 \%)\end{array}$ & $2(33.3 \%)$ & $\begin{array}{l}4 \\
(66.7 \%)\end{array}$ & - & - & \\
\hline Wan & ge & $8(88.9 \%)$ & $\begin{array}{l}8 \\
(100.0 \%)\end{array}$ & & & & . & $\begin{array}{l}1 \\
(11.1 \%)\end{array}$ & $\begin{array}{l}1 \\
(100.0 \%)\end{array}$ & & & & \\
\hline $\begin{array}{l}\text { Huang, } \\
\text { Wang }\end{array}$ & $3^{\circ}$ & $3(100.0 \%)$ & $\begin{array}{l}3 \\
(100.0 \%)\end{array}$ & - & - & - & . & $0(0.0 \%)$ & $0(0.0 \%)$ & - & & - & \\
\hline Rentsch & 285 & $\begin{array}{l}168 \\
(58.9 \%)^{*}\end{array}$ & $\begin{array}{l}47 \\
(28.0 \%)\end{array}$ & $\begin{array}{l}53 \\
(31.5 \%)\end{array}$ & - & $\begin{array}{l}68 \\
(40.4 \%)\end{array}$ & . & $\begin{array}{l}117 \\
(21.1 \%)\end{array}$ & $\begin{array}{l}43 \\
(36.8 \%)\end{array}$ & $\begin{array}{l}36 \\
(30.8 \%)\end{array}$ & - & $\begin{array}{l}38 \\
(32.5 \%)\end{array}$ & \\
\hline Hu & 323 & $\begin{array}{l}151 \\
(46.7 \%)\end{array}$ & - & - & $12(7.9 \%)$ & - & $139(92.1 \%)$ & $\begin{array}{l}172 \\
(53.3 \%)\end{array}$ & - & - & $26(15.1 \%)$ & - & $146(84.9 \%)$ \\
\hline Wang & 125 & $\begin{array}{l}100 \\
(80.0 \%)\end{array}$ & & & $9(9.0 \%)$ & & 91 (91.0\%) & $\begin{array}{l}25 \\
(20.0 \%)\end{array}$ & & & $7(28.0 \%)$ & & $18(72.0 \%)$ \\
\hline Petrilli & 4103 & $\begin{array}{l}932 \\
(22.7 \%)^{*}\end{array}$ & $62(6.7 \%)$ & $\begin{array}{l}175 \\
(18.8 \%)\end{array}$ & - & & 695 (74.6\%) & $\begin{array}{l}650 \\
(15.8 \%)\end{array}$ & $28(4.3 \%)$ & $\begin{array}{l}145 \\
(22.3 \%)\end{array}$ & & - & $477(73.4 \%)$ \\
\hline Shi & $474^{\circ}$ & $\begin{array}{l}425 \\
(89.7 \%)\end{array}$ & - & - & $34(8.0 \%)$ & - & $391(92.0 \%)$ & $\begin{array}{l}49 \\
(10.3 \%)\end{array}$ & - & - & $6(12.2 \%)$ & - & $43(87.8 \%)$ \\
\hline Kim & $27^{\ddagger}$ & $21(77.7 \%)$ & $3(14.3 \%)$ & - & - & - & $18(85.7 \%)$ & $\begin{array}{l}\text { (22.2\%) } \\
\text { (1) }\end{array}$ & 2 (33.3\%) & - & - & - & $4(66.6 \%)$ \\
\hline
\end{tabular}

Note. ${ }^{a}$ Data on smoking status were missing for 14 participants; ${ }^{b}$ Data on smoking status were missing for 131 participants; ${ }^{\mathrm{D}}$ Data on smoking status were missing for 126 participants; ${ }^{\mathrm{d}}$ Data on smoking status were missing for 38 participants; ${ }^{\mathrm{e}}$ Data on smoking status were missing for 13 participants; ${ }^{\mathrm{f}}$ Data on smoking status were missing for 1 participant; * Patients with disease requiring hospital (but not ITU) admission

Table 5. Mortality by smoking status.

\begin{tabular}{|c|c|c|c|c|c|c|c|c|c|c|c|}
\hline & $\begin{array}{l}\text { Sample } \\
\text { size }\end{array}$ & Death & & & & & Recovery & & & & \\
\hline Author & $\mathrm{N}$ & $\mathrm{n}$ & $\begin{array}{l}\text { Current } \\
\text { smoker }\end{array}$ & $\begin{array}{l}\text { Former } \\
\text { smoker }\end{array}$ & $\begin{array}{l}\text { Never } \\
\text { smoker }\end{array}$ & $\begin{array}{l}\text { Never } \\
\text { smoker/unknown }\end{array}$ & $n$ & $\begin{array}{l}\text { Current } \\
\text { smoker }\end{array}$ & $\begin{array}{l}\text { Former } \\
\text { smoker }\end{array}$ & $\begin{array}{l}\text { Never } \\
\text { smoker }\end{array}$ & $\begin{array}{l}\text { Never } \\
\text { smoker/unknown }\end{array}$ \\
\hline Chen & $274^{*}$ & $\begin{array}{l}113 \\
(41.2 \%)\end{array}$ & $7(6.2 \%)$ & $2(1.8 \%)$ & - & $104(92.0 \%)^{\wedge}$ & $161(58.8)$ & $5(3.1 \%)$ & - & - & 156 (96.9\%) \\
\hline Zhou & 191 & $\begin{array}{l}54 \\
(28.3 \%)\end{array}$ & $5(9.3 \%)$ & - & - & $49(90.7 \%)$ & $\begin{array}{l}137 \\
(71.7 \%)\end{array}$ & $6(4.4 \%)$ & - & - & 131 (95.6\%) \\
\hline Yang & 52 & $\begin{array}{l}32 \\
(61.5 \%)\end{array}$ & $0(0.0 \%)$ & - & - & $32(100.0 \%)$ & 20 & $2(10.0 \%)$ & - & - & $18(90.0 \%)$ \\
\hline
\end{tabular}

Note. * Data on mortality were missing for 274 participants; ^ No smoking history defined as <30 pack-years of smoking

\section{Discussion}

This rapid evidence review of 28 observational studies found substantial uncertainty arising from the recording of smoking status. There was generally lower than expected smoking rates in the studied populations compared with national smoking prevalence, but the comparisons include 25 studies which did not explicitly record or report on never smoking status. From available data, there was insufficient evidence to establish whether current and/or former smoking status is associated with SARS-CoV-2 infection, hospitalisation or mortality. There was low quality evidence that disease severity in those hospitalised for COVID-19 is greater in current/former smokers compared with never smokers.

\section{Infection by smoking status}

Current data suggest that smokers in the community appear to be less likely to test positive for SARS-CoV-2 compared with never smokers. It should, however, be noted 
that smokers were tested at a higher rate than never smokers in the only study available. Smokers may be more likely to meet local criteria for community testing due to increased prevalence of symptoms consistent with SARS-CoV-2 infection, such as cough and increased sputum production. We would therefore caution against drawing any conclusion as to whether smokers are at increased risk of SARS-CoV-2 infection at this early stage.

\section{Hospitalisation and disease severity by smoking status}

There was no evidence that current/former smoker are at greater risk of hospitalisation for COVID-19. However, these early studies are limited by several factors. First, they primarily describe populations admitted to hospital in the context of an emerging epidemic; hence, it is likely that data collection on smoking status was not considered a priority. As a consequence, current smoking rates are likely to be underreported and former smoking may have been conflated with never smoking. In China, approximately $69 \%$ of men aged $60+$ years have a history of or are current smokers, which suggests that a non-trivial proportion of former smokers for whom a period of regular smoking earlier in life might have caused lasting disruption to the respiratory system have not been taken into account in the included studies. Second, individuals with severe symptoms from COVID-19 may have stopped smoking prior to admission to a care facility and may therefore not have been recorded as current smokers (i.e. reverse causality). Third, smokers with COVID-19 may be less likely to present to hospital because of lack of access to healthcare and more likely to die in the community from sudden complications (i.e. self-selection). Fourth, it should also be noted that smoking is a risk factor for both hypertension and diabetes, two diseases associated with worse outcomes from COVID-19, which suggests that current and former smoking may be both directly and indirectly implicated in COVID-19 outcomes. Last, reason for hospitalisation varies by country and time in the epidemic. For example, initial cases may have been hospitalised for isolation and quarantine reasons and not due to medical necessity. It is plausible that this may have skewed early data towards less severe cases.

\section{Mortality by smoking status}

Although there is currently insufficient evidence as to whether current/former smokers are at greater risk of death from COVID-19, it should be noted that these early studies have not followed all patients for a sufficient period of time to report such an outcome. 


\section{Implications for research, policy and practice}

We believe that high quality smoking cessation advice should form part of public health efforts during COVID-19. At the very least, smoking cessation reduces acute risks from cardiovascular disease and could reduce demands on the healthcare system ${ }^{[53]}$. GPs and other healthcare providers can play a crucial role - brief, high-quality and free online training is available from NCSCT.

\section{Conclusion}

Across 28 observational studies, there is substantial uncertainty arising from the recording of smoking status on whether current and/or former smoking status is associated with SARS-CoV-2 infection, hospitalisation or mortality. There is low quality evidence that current and former smoking compared with never is associated with greater disease severity in those hospitalised for COVID-19.

\section{Acknowledgements}

JB and OP receive salary support from Cancer Research UK (C1417/A22962). LS, JB and OP are members of SPECT RUM, a UK Prevention Research Partnership Consortium (MR/S037519/1). UKPRP is an initiative funded by the UK Research and Innovation Councils, the Department of Health and Social Care (England) and the UK devolved administrations, and leading health research charities.

\section{Conflicts of interest}

DS and OP report no conflicts of interest. LS has received a research grant and honoraria for a talk and travel expenses from manufacturers of smoking cessation medications (Pfizer and Johnson \& Johnson). JB has received unrestricted research funding from Pfizer to study smoking cessation.

\section{References}

1. a, b Wei-jie Guan, Zheng-yi Ni, Yu Hu, Wen-hua Liang, Chun-quan Ou, Jian-xing He. (2020). Clinical Characteristics of Coronavirus Disease 2019 in China. N EnglJ Med. doi:10.1056/nejmoa2002032.

2. ^Markus Hoffmann, Hannah Kleine-Weber, Simon Schroeder, Nadine Krüger, Tanja 
Herrler, Sandra Erichsen. (2020). SARS-CoV-2 Cell Entry Depends on ACE2 and

TMPRSS2 and Is Blocked by a Clinically Proven Protease Inhibitor. Cell, vol. 181 (2),

271-280.e8. doi:10.1016/j.cell.2020.02.052.

3. ^Samuel James Brake, Kathryn Barnsley, Wenying Lu, Kielan Darcy McAlinden, Mathew Suji Eapen, Sukhwinder Singh Sohal. (2020). Smoking Upregulates AngiotensinConverting Enzyme-2 Receptor: A Potential Adhesion Site for Novel Coronavirus SARS-CoV-2 (Covid-19). JCM, vol. 9 (3), 841. doi:10.3390/jcm9030841.

4. ^ Guoshuai Cai. (2020). Bulk and Single-Cell Transcriptomics Identify Tobacco-Use Disparity in Lung Gene Expression of ACE2, the Receptor of 2019-nCov. doi:10.20944/preprints202002.0051.v3.

5. ^ Joshua M. Oakes, Robert M. Fuchs, Jason D. Gardner, Eric Lazartigues, Xinping Yue. (2018). Nicotine and the renin-angiotensin system. American Journal of PhysiologyRegulatory, Integrative and Comparative Physiology, vol. 315 (5), R895-R906. doi:10.1152/ajpregu.00099.2018.

6. ' Xiuming Xi, the Chinese Critical Care Clinical Trial Group (CCCCTG), Yuan Xu, Li Jiang, Ang Li, Jie Duan. (2010). Hospitalized adult patients with 2009 influenza A(H1N1) in Beijing, China: risk factors for hospital mortality. BMC Infect Dis, vol. 10 (1). doi:10.1186/1471-2334-10-256.

7. ^ Tochukwu RaphaelAbadom, Adrian D. Smith, Stefano Tempia, Shabir A. Madhi, Cheryl Cohen, Adam L. Cohen. (2016). Risk factors associated with hospitalisation for influenza-associated severe acute respiratory illness in South Africa: A case-population study. Vaccine, vol. 34 (46), 5649-5655. doi:10.1016/j.vaccine.2016.09.011.

8. ^ Jordi Almirall, Carlos A. González, Xavier Balanzó, Ignasi Bolibar. (1999). Proportion of Community-Acquired Pneumonia Cases Attributable to Tobacco Smoking. Chest, vol. 116 (2), 375-379. doi:10.1378/chest.116.2.375.

9. ^ Charles Feldman, Ronald Anderson. (2013). Cigarette smoking and mechanisms of susceptibility to infections of the respiratory tract and other organ systems. Journal of Infection, vol. 67 (3), 169-184. dol:10.1016/j.jinf.2013.05.004.

10. ^ JA Dye, K B Adler. (1994). Effects of cigarette smoke on epithelial cells of the respiratory tract.. Thorax, vol. 49 (8), 825-834. doi:10.1136/thx.49.8.825.

11. ^ Constantine Vardavas, Katerina Nikitara. (2020). COVID-19 and smoking: A systematic review of the evidence. Tob. Induc. Dis., vol. 18 (March). doi:10.18332/tid/119324.

12. ^Emami et al.. (2020). Prevalence of Underlying Diseases in Hospitalized Patients with COVID-19: a Systematic Review and Meta-Analysis. Archives of Academic Emergency 
Medicine, vol. 8 .

13. ^ Jaber S Alqahtani, Tope Oyelade, Abdulelah M Aldhahir, Saeed M Alghamdi, Mater Almehmadi, Abdullah S Alqahtani. (2020). Prevalence, Severity and Mortality associated with COPD and Smoking in patients with COVID-19: A Rapid Systematic Review and Meta-Analysis. doi:10.1101/2020.03.25.20043745.

14. ^ Roengrudee Patanavanich, Stanton A Glantz. (2020). Smoking is Associated with COVID-19 Progression: A Meta-Analysis. doi:10.1101/2020.04.13.20063669.

15. ^ Konstantinos Farsalinos, Anastasia Barbouni, Raymond Niaura. (2020). Smoking, vaping and hospitalization for COVID-19. Qeios. doi:10.32388/269o8a.13.

16. ^Ivan Berlin, Daniel Thomas, Anne-Laurence Le Faou, Jacques Cornuz. (2020). COVID19 and Smoking. doi:10.1093/ntr/ntaa059.

17. ^ Andrea C. Tricco, Jesmin Antony, Wasifa Zarin, Lisa Strifler, Marco Ghassemi, John Ivory. (2015). A scoping review of rapid review methods. BMC Med, vol. 13 (1). doi:10.1186/s12916-015-0465-6.

18. ^ The Cochrane Collaboration. (2014). Review Manager (RevMan). Version 5.3. Copenhagen: The Nordic Cochrane Centre.

19. ^Julian P. T. Higgins, Sally Green. (2011). Cochrane Handbook for Systematic Reviews of Interventions. John Wiley \& Sons.

20. ^J. P T Higgins. (2003). Measuring inconsistency in meta-analyses. BMJ, vol. 327 (7414), 557-560. doi:10.1136/bmj.327.7414.557.

21. ^ Wei-jie Guan, Wen-hua Liang, Yi Zhao, Heng-rui Liang, Zi-sheng Chen, Yi-min Li. (2020). Comorbidity and its impact on 1590 patients with Covid-19 in China: A Nationwide Analysis. Eur Respir J. doi:10.1183/13993003.00547-2020.

22. ^ Xiaoli Zhang, Huan Cai, Jianhua Hu, Jiangshan Lian, Jueqing Gu, Shanyan Zhang. (2020). Epidemiological, clinical characteristics of cases of SARS-CoV-2 infection with abnormal imaging findings. International Journal of Infectious Diseases, vol. 94 , 81-87. doi:10.1016/j.jijd.2020.03.040.

23. ^ Chaolin Huang, Yeming Wang, Xingwang Li, Lili Ren, Jianping Zhao, Yi Hu. (2020). Clinical features of patients infected with 2019 novel coronavirus in Wuhan, China. The Lancet, vol. 395 (10223), 497-506. doi:10.1016/s0140-6736(20)30183-5.

24. ^ Ru Liu, Xiaoyan Ming, Ou Xu, Jianli Zhou, Hui Peng, Ning Xiang. (2020). Association of Cardiovascular Manifestations with In-hospital Outcomes in Patients with COVID-19: A Hospital Staff Data. doi:10.1101/2020.02.29.20029348.

25. ^ Tao Guo, Yongzhen Fan, Ming Chen, Xiaoyan Wu, Lin Zhang, Tao He. (2020). Cardiovascular Implications of Fatal Outcomes of Patients With Coronavirus Disease 
2019(COVID-19). JAMA Cardiol. doi:10.1001/jamacardio.2020.1017.

26. ^ Huayan Xu, Keke Hou, Hong Xu, Zhenlin Li, Huizhu Chen, Na Zhang. (2020). Acute Myocardial Injury of Patients with Coronavirus Disease 2019. doi:10.1101/2020.03.05.20031591.

27. ^ Ying Huang, Rui Yang, Ying Xu, Ping Gong. (2020). Clinical characteristics of 36 nonsurvivors with COVID-19 in Wuhan, China. doi:10.1101/2020.02.27.20029009.

28. ` Ling Hu, Shaoqiu Chen, Yuanyuan Fu, Zitong Gao, Hui Long, Hong-wei Ren. (2020). Risk Factors Associated with Clinical Outcomes in 323 COVID-19 Patients in Wuhan, China. doi:10.1101/2020.03.25.20037721.

29. ^ Ruirui Wang, Min Pan, Xiumei Zhang, Xiaoyun Fan, Mingfeng Han, Fengde Zhao. (2020). Epidemiological and clinical features of 125 Hospitalized Patients with COVID19 in Fuyang, Anhui, China. International Journal of Infectious Diseases. doi:10.1016/j.ijid.2020.03.070.

30. ^ Jiangshan Lian, XiJin, Shaorui Hao, Huan Cai, Shanyan Zhang, Lin Zheng. (2020). Analysis of Epidemiological and Clinical features in older patients with Corona Virus Disease 2019 (COVID-19) out of Wuhan. doi:10.1093/cid/ciaa242.

31. ^ FeiZhou, Ting Yu, Ronghui Du, Guohui Fan, Ying Liu, Zhibo Liu. (2020). Clinical course and risk factors for mortality of adult inpatients with COVID-19 in Wuhan, China: a retrospective cohort study. The Lancet, vol. 395 (10229), 1054-1062. doi:10.1016/s0140-6736(20)30566-3.

32. a, b Pingzheng Mo, Yuanyuan Xing, Yu Xiao, Liping Deng, Qiu Zhao, Hongling Wang. (2020). Clinical characteristics of refractory COVID-19 pneumonia in Wuhan, China. doi:10.1093/cid/ciaa270.

33. a, b Tao Chen, Di Wu, Huilong Chen, Weiming Yan, Danlei Yang, Guang Chen. (2020). Clinical characteristics of 113 deceased patients with coronavirus disease 2019: retrospective study. BMJ. doi:10.1136/bmj.m1091.

34. `Jin-jin Zhang, Xiang Dong, Yi-yuan Cao, Ya-dong Yuan, Yi-bin Yang, You-qin Yan. (2020). Clinical characteristics of 140 patients infected with SARS-COV-2 in Wuhan, China. Allergy. doi:10.1111/all.14238.

35. ^ Suxin Wan, YiXiang, Wei Fang, Yu Zheng, Boqun Li, Yanjun Hu. (2020). Clinical features and treatment of COVID-19 patients in northeast Chongqing.J Med Virol. doi:10.1002/jmv.25783.

36. ^ Xi Jin, Jiang-Shan Lian, Jian-Hua Hu, Jianguo Gao, Lin Zheng, Yi-Min Zhang. (2020). Epidemiological, clinical and virological characteristics of 74 cases of coronavirusinfected disease 2019 (COVID-19) with gastrointestinal symptoms. Gut. 
doi:10.1136/gutjnl-2020-320926.

37. ${ }^{a}$ b Wei Liu, Zhao-Wu Tao, Wang Lei, Yuan Ming-Li, Liu Kui, Zhou Ling. (2020). Analysis of factors associated with disease outcomes in hospitalized patients with 2019 novel coronavirus disease. Chinese MedicalJournal. doi:10.1097/cm9.0000000000000775.

38. a, b Christopher T. Rentsch, Farah Kidwai-Khan, Janet P. Tate, Lesley S. Park, Joseph T. King, Melissa Skanderson. (2020). Covid-19 Testing, Hospital Admission, and Intensive Care Among 2,026,227 United States Veterans Aged 54-75 Years. doi:10.1101/2020.04.09.20059964.

39. a, b Christopher M. Petrilli, Simon A. Jones, Jie Yang, Harish Rajagopalan, Luke F. O'Donnell, Yelena Chernyak. (2020). Factors associated with hospitalization and critical illness among 4,103 patients with COVID-19 disease in New York City. doi:10.1101/2020.04.08.20057794.

40. a, b CDC COVID-19 Response Team, Nancy Chow, Katherine Fleming-Dutra, Ryan Gierke, Aron Hall, Michelle Hughes. (2020). Preliminary Estimates of the Prevalence of Selected Underlying Health Conditions Among Patients with Coronavirus Disease 2019 - United States, February 12-March 28, 2020. MMWR Morb. Mortal. Wkly. Rep., vol. 69 (13), 382-386. doi:10.15585/mmwr.mm6913e2.

41. ^ Eu Suk Kim, Bum Sik Chin, Chang Kyung Kang, Nam Joong Kim, Yu Min Kang, Jae-Phil Choi. (2020). Clinical Course and Outcomes of Patients with Severe Acute Respiratory Syndrome Coronavirus 2 Infection: a Preliminary Report of the First 28 Patients from the Korean Cohort Study on COVID-19.J Korean Med Sci, vol. 35 (13). doi:10.3346/jkms.2020.35.e142.

42. a, b, c Makoto Miyara, Florence Tubach, Zahir Amoura. (2020). Low incidence of daily active tobacco smoking in patients with symptomatic COVID-19 infection. Qeios. doi:10.32388/wpp19w.

43. ^ ISARIC. (2020). International Severe Acute Respiratory and Emerging Infection Consortium.

44. ^ Yu Shi, Xia Yu, Hong Zhao, Hao Wang, Ruihong Zhao, Jifang Sheng. (2020). Host susceptibility to severe COVID-19 and establishment of a host risk score: findings of 487 cases outside Wuhan. Crit Care, vol. 24 (1). doi:10.1186/s13054-020-2833-7.

45. ^Xiaobo Yang, Yuan Yu, Jiqian Xu, Huaqing Shu, Jia'an Xia, Hong Liu. (2020). Clinical course and outcomes of critically ill patients with SARS-COV-2 pneumonia in Wuhan, China: a single-centered, retrospective, observational study. The Lancet Respiratory Medicine. doi:10.1016/s2213-2600(20)30079-5.

46. a, b Xiang Dong, Yi-yuan Cao, Xiao-xia Lu, Jin-jin Zhang, Hui Du, You-qin Yan. (2020). 
Eleven faces of coronavirus disease 2019. Allergy. doi:10.1111/all.14289.

47. ^ Chinese Center for Disease Control and Prevention. (2018). Global Adult Tobacco Survey, China 2018..

48. ^ Center for Health Statistics. Table A-12. Current cigarette smoking status among adults aged 18 and over, by selected characteristics: United States, 2018.

49. `Y Youngs Chang, Hee-Yeon Kang, Dohee Lim, Hong-Jun Cho, Young-Ho Khang. (2019). Long-term trends in smoking prevalence and its socioeconomic inequalities in Korea, 1992-2016. Int J Equity Health, vol. 18(1). doi:10.1186/s12939-019-1051-x.

50. ^ Richard et al.. (2019). Baisse de la prévalence du tabagisme quotidien parmi les adultes : résultats du Baromètre de Santé publique France 2018. Bull Epidémiol Hebd.

51. ^ ONS. (2019). Smoking habits in the UK and its constituent countries..

52. `Satomi Odani, Israel T. Agaku, Corinne M. Graffunder, MichaelA. Tynan, Brian S. Armour. (2018). Tobacco Product Use Among Military Veterans - United States, 2010-2015. MMWR Morb. Mortal. Wkly. Rep., vol. 67 (1), 7-12. doi:10.15585/mmwr.mm6701a2.

53. ^ Lindsay F Stead, Diana Buitrago, Nataly Preciado, Guillermo Sanchez, Jamie Hartmann-Boyce, Tim Lancaster. (2013). Physician advice for smoking cessation. doi:10.1002/14651858.cd000165.pub4. 